PROCEEDINGS OF THE AMERICAN MATHEMATICAL SOCIETY

Volume 125, Number 4, April 1997, Pages 979-985

S 0002-9939(97)03678-2

\title{
ON PERFECT SIMPLE-INJECTIVE RINGS
}

\author{
W. K. NICHOLSON AND M. F. YOUSIF
}

(Communicated by Ken Goodearl)

Dedicated to Professor K. Varadarajan on the occasion of his sixtieth birthday

\begin{abstract}
Harada calls a ring $R$ right simple-injective if every $R$-homomorphism with simple image from a right ideal of $R$ to $R$ is given by left multiplication by an element of $R$. In this paper we show that every left perfect, left and right simple-injective ring is quasi-Frobenius, extending a well known result of Osofsky on self-injective rings. It is also shown that if $R$ is left perfect and right simple-injective, then $R$ is quasi-Frobenius if and only if the second socle of $R$ is countably generated as a left $R$-module, extending many recent results on self-injective rings. Examples are given to show that our results are non-trivial extensions of those on self-injective rings.
\end{abstract}

A ring $R$ is called quasi-Frobenius if $R$ is left (and right) artinian and left (and right) self-injective. A well known result of Osofsky [15] asserts that a left perfect, left and right self-injective ring is quasi-Frobenius. It has been conjectured by Faith [9] that a left (or right) perfect, right self-injective ring is quasi-Frobenius. This conjecture remains open even for semiprimary rings.

Throughout this paper all rings $R$ considered are associative with unity and all modules are unitary $R$-modules. We write $M_{R}$ to indicate a right $R$-module. The socle of a module is denoted by $\operatorname{soc}(M)$. We write $N \subseteq M\left(N \subseteq \subseteq^{\text {ess }} M\right)$ to mean that $N$ is a submodule (essential) of $M$. For any subset $X$ of $R, l(X)$ and $r(X)$ denote, respectively, the left and right annihilators of $X$ in $R$.

A ring $R$ is called right Kasch if every simple right $R$-module is isomorphic to a minimal right ideal of $R$. The ring $R$ is called right pseudo-Frobenius (a right PF-ring) if $R_{R}$ is an injective cogenerator in mod- $R$; equivalently if $R$ is semiperfect, right self-injective and has an essential right socle.

A ring $R$ is called right principally injective if every $R$-morphism from a principal right ideal of $R$ into $R$ is given by left multiplication. In [14], a ring $R$ is called a right generalized pseudo-Frobenius ring (a right GPF-ring) if $R$ is semiperfect, right principally injective and has an essential right socle.

We write $J=J(R)$ for the Jacobson radical of the ring $R$. Following Fuller [10], if $R$ is semiperfect with a basic set $E$ of primitive idempotents, and if $e, f \in E$, we say that the pair $(e R, R f)$ is an i-pair if $\operatorname{soc}(e R) \cong f R / f J$ and $\operatorname{soc}(R f) \cong R e / J e$.

Received by the editors April 24, 1995 and, in revised form, October 11, 1995.

1991 Mathematics Subject Classification. Primary 16D50, 16L30.

Key words and phrases. Perfect ring, self-injective ring, quasi-Frobenius ring.

The research of both authors was supported by NSERC Grant 8075 and by the Ohio State University.

(C) 1997 American Mathematical Society 
Let $M_{R}$ and $N_{R}$ be $R$-modules. Following Harada [12], $M$ is said to be a simpleN-injective module if, for any submodule $X \subseteq N$ and any $R$-morphism $\gamma: X \rightarrow$ $M$ such that $\operatorname{im}(\gamma)$ is simple, there exists an $R$-morphism $\hat{\gamma}: N \rightarrow M$ such that $\left.\hat{\gamma}\right|_{X}=\gamma$. We call a ring $R$ right simple-injective if $R_{R}$ is simple- $R$-injective; equivalently, if $I$ is a right ideal of $R$ and $\gamma: I \rightarrow R$ is an $R$-morphism with simple image, then $\gamma=c$. is left multiplication by an element $c \in R$.

If $M$ is a right $R$-module, $\operatorname{soc}_{\alpha}(M)$ is defined for each ordinal $\alpha$ as follows: (1) $\operatorname{soc}_{1}(M)=\operatorname{soc}(M) ;(2)$ If $\operatorname{soc}_{\alpha}(M)$ has been defined, then $\operatorname{soc}_{\alpha+1}(M)$ is given by $\operatorname{soc}\left[M / \operatorname{soc}_{\alpha}(M)\right]=\operatorname{soc}_{\alpha+1}(M) / \operatorname{soc}_{\alpha}(M)$; (3) If $\alpha$ is a limit ordinal, then $\operatorname{soc}_{\alpha}(M)=\bigcup_{\beta<\alpha} \operatorname{soc}_{\beta}(M)$. The series $\operatorname{soc}_{1}(M) \subseteq \operatorname{soc}_{2}(M) \subseteq \cdots$ is called the Lowey series of the module $M$.

Motivated by the work of Armendariz and Park [2] and that of Ara and Park [1], it was shown by Clark and Huynh [8] that if $R$ is a left and right perfect, right self-injective ring, then $R$ is quasi-Frobenius if and only if $\operatorname{soc}_{2}(R)$ is finitely generated as a right $R$-module. The authors did not indicate whether their result remains valid if $\operatorname{soc}_{2}(R)$ is finitely generated as a left $R$-module. However it is not difficult to see that if $R$ is left perfect and right self-injective, and if $\operatorname{soc}_{2}(R)$ is finitely generated as a left $R$-module, then $R$ is quasi-Frobenius. Indeed, since $R$ is a right PF-ring, $l(J)=r(J)=\operatorname{soc}\left({ }_{R} R\right)=\operatorname{soc}\left(R_{R}\right)$, and hence $l\left(J^{2}\right)=r\left(J^{2}\right)=$ $\operatorname{soc}_{2}\left(R_{R} R\right)=\operatorname{soc}_{2}\left(R_{R}\right)$. Suppose $\operatorname{soc}_{2}(R)=\sum_{i=1}^{n} R a_{i}, a_{i} \in R$, and define $\varphi: R \rightarrow$ $\bigoplus_{i=1}^{n} a_{i} R$ by $\varphi(r)=\left(a_{1} r, \ldots, a_{n} r\right)$. Then $\operatorname{ker} \varphi=r\left(\operatorname{soc}_{2}(R)\right)=r l\left(J^{2}\right)=J^{2}$. Thus $R / J^{2} \hookrightarrow \bigoplus_{i=1}^{n} a_{i} R$ and it follows that $J / J^{2}$ is right finite dimensional (because $R$ is a right PF-ring). Hence $J / J^{2}$ is right finitely generated, and so $R$ is right artinian by a result of Osofsky [15]. This observation implies several results in the literature (see for example [2]) and will be generalized in Theorem 2 of this paper.

The following two lemmas will be needed in our investigation.

Lemma 1 ([14], Theorem 2.3). Let $R$ be a right GPF-ring. If $\left\{e_{1}, \ldots, e_{n}\right\}$ is a basic set of primitive idempotents, there exists a (Nakayama) permutation $\sigma$ of $\left\{e_{1}, \ldots, e_{n}\right\}$ such that, for each $k=1,2, \ldots, n, \operatorname{soc}\left(R e_{\sigma k}\right) \cong R e_{k} / J e_{k}$ is essential in $R e_{\sigma k}$ and $\operatorname{soc}\left(e_{k} R\right)$ is homogeneous with each simple submodule isomorphic to $e_{\sigma k} R / e_{\sigma k} J$. We also have $\operatorname{soc}\left({ }_{R} R\right)=\operatorname{soc}\left(R_{R}\right)$.

The statement of Proposition 2 in [3] includes the hypothesis that $(e R, R f)$ is an i-pair. However this hypothesis is not used in the proof, so the result should be stated as follows:

Lemma 2 ([3], Proposition 2). Let $R$ be a semiprimary ring and let $E$ be a basic set of primitive idempotents in $R$. If $e \in E$ and $e R$ is simple-gR-injective for every $g \in E$, then $e R$ is injective.

Proposition 1. Suppose $R$ is semiperfect with a basic set $\left\{e_{1}, e_{2}, \ldots, e_{n}\right\}$ of primitive idempotents. If $R$ is right simple-injective and $\operatorname{soc}\left(R_{R}\right) \subseteq \subseteq^{\mathrm{ess}} R_{R}$, there exists a (Nakayama) permutation $\sigma$ of $\{1,2, \ldots, n\}$ such that $\left(e_{k} R, R e_{\sigma k}\right)$ is an $i$-pair for each $k=1,2, \ldots, n$.

Proof. The proof is divided into separate claims.

Claim 1. $R$ is right Kasch.

Proof. For each $i$ choose a simple right ideal $K_{i} \subseteq e_{i} R$. It suffices to show that the $K_{i}$ are a set of distinct representatives of the simple right $R$-modules. Since the $e_{i}$ are basic, we show that $K_{i} \cong K_{j}$ implies $e_{i} R \cong e_{j} R$. But if $\sigma: K_{i} \rightarrow K_{j}$ is an 
isomorphism, then $\sigma=a \cdot, a \in R$, because $R$ is right simple-injective. If $\sigma^{-1}=b$. and $K_{i}=k R$, then $b a k=k$ and it follows that $a \notin J$. Since we may assume $a \in e_{j} R e_{i}$, it follows that $a \cdot: e_{i} R \rightarrow e_{j} R$ is an isomorphism, proving Claim 1.

Claim 2. $\operatorname{rl}(I)=I$ for every right ideal $I$ of $R$.

Proof. If $b \in \operatorname{rl}(I), b \notin I$, let $M / I$ be a maximal submodule of $(b R+I) / I$. If $\delta:(b R+I) / M \rightarrow R_{R}$ is an embedding (by Claim 1$)$, define $\gamma:(b R+I) \rightarrow R$ by $\gamma(x)=\delta(x+M)$. Then $\gamma=c$. for some $c \in R$. Thus $c I=\gamma(I)=0$, so $c b=0$ because $b \in \operatorname{rl}(I)$. But $c b=\delta(b+M) \neq 0$, proving Claim 2 .

In particular, $R$ is left principally injective because $r l(a)=a R$ for all $a \in R$. For convenience, write $S_{r}=\operatorname{soc}\left(R_{R}\right)$ and $S_{l}=\operatorname{soc}\left({ }_{R} R\right)$.

Claim 3. $S_{r} \subseteq{ }_{R}^{\text {ess }} R$. In particular $S_{l} \subseteq S_{r}$.

Proof. Let $0 \neq b \in R$. As in Claim 2, let $\gamma: b R \rightarrow R_{R}$ have simple image. Then $\gamma=c$. for $c \in R$, so $c b=\gamma(b) \neq 0$, while $c b J \subseteq \gamma(b R) J=0$. Thus $0 \neq c b \in R b \cap l(J)$. Since $l(J)=S_{r}(R / J$ is semisimple), this proves Claim 3 .

Claim 4. If $k R$ is simple, $k \in R$, then $R k=\operatorname{lr}(k)$ is simple. In particular $S_{l}=S_{r}$.

Proof. Let $k R$ be simple, $k \in R$. If $0 \neq a \in R k$, then $r(k) \subseteq r(a)$ so $r(k)=r(a)$ because $r(k)$ is maximal. Hence $k \in \operatorname{lr}(k)=\operatorname{lr}(a)$ and it suffices to show that $\operatorname{lr}(a) \subseteq R a$. But if $b \in \operatorname{lr}(a)$, then $\gamma: a R \rightarrow R$ is well defined by $\gamma(a r)=b r$. As $a R$ is simple, $\gamma=c \cdot c \in R$, so $b=\gamma(a)=c a \in R a$, proving Claim 4 .

Thus $S_{l}$ is essential as a left ideal (by Claims 3 and 4 ) so, since $R$ is semiperfect and left principally injective, we conclude that $R$ is a left GPF-ring.

Claim 5. If $R k$ is simple, $k \in R$, then $k R$ is simple.

Proof. If $R k$ is simple, then $k R \subseteq S_{r}$ by Claim 4, so let $k R \supseteq m R, m R$ simple. Then $l(k) \subseteq l(m)$ so $l(k)=l(m)$ (as $l(k)$ is maximal). But then $k R=m R$ by Claim 2, proving Claim 5.

Claim 6. $\operatorname{soc}(R e)$ is simple for every primitive idempotent $e \in R$.

Proof. We have $S_{l} \subseteq{ }^{\text {ess }}{ }_{R} R$ by Claims 3 and 4 , so let $R k \subseteq \operatorname{soc}(R e), R k$ is a simple left ideal. Then $r(k) \supseteq(1-e) R+J$ using Claim 5 . But $(1-e) R+J$ is a maximal right ideal ( $e$ is local), whence $r(k)=(1-e) R+J$. Thus, using Claim 4, $R k=\operatorname{lr}(k)=\operatorname{Re} \cap l(J)=\operatorname{Re} \cap S_{r}=\operatorname{soc}(R e)$. This proves Claim 6 .

Now, since $R$ is a left GPF-ring, it follows from Lemma 1 and Claim 6 that there is a permutation $\sigma$ of $\{1,2, \ldots, n\}$ such that $\operatorname{soc}\left(e_{k} R\right) \cong e_{\sigma k} R / e_{\sigma k} J$ and $\operatorname{soc}\left(R e_{\sigma k}\right) \cong R e_{k} / J e_{k}$ for each $k$. Hence $\left(e_{k} R, R e_{\sigma k}\right)$ is an i-pair for each $k=$ $1,2, \ldots, n$. This proves Proposition 1 .

The next proposition is now an easy consequence of Lemma 2 .

Proposition 2. Let $R$ be a semiprimary ring. Then $R$ is right self-injective if and only if $R$ is right simple-injective.

Proof. It is routine to see that if $R$ is right simple-injective, then $e R$ is simple$g R$-injective for all primitive idempotents $e$ and $g$. Hence each $e R$ is injective by Proposition 1 and Lemma 2 , so $R_{R}$ is injective. 
Recall that $S_{r} \subseteq{ }^{\text {ess }} R_{R}$ whenever $R$ is left perfect. Hence the next result extends a well known theorem of Osofsky [15] which states that a left perfect, left and right self-injective ring is quasi-Frobenius.

Proposition 3. Suppose $R$ is a left perfect, left and right simple-injective ring. Then $R$ is a quasi-Frobenius ring.

Proof. From the proof of Proposition 1, it follows that $R$ is a left GPF-ring in which $r l(I)=I$ for every right ideal $I$ of $R$ and $\operatorname{soc}\left({ }_{R} R\right)=\operatorname{soc}\left(R_{R}\right) \subseteq{ }^{\text {ess }}{ }_{R} R$. By symmetry, $\operatorname{lr}(L)=L$ for every left ideal $L$ of $R$. Now [11, Theorem 5.3] shows that every principal left or right $R$-module has finite uniform dimension. As $R$ is right semiartinian ( $R$ is left perfect), it follows that $R$ is right artinian [4, Proposition 5]. In particular, $R$ is semiprimary. Thus Proposition 2 implies that $R$ is left and right self-injective. Whence $R$ is quasi-Frobenius.

The original proof of Proposition 4 below was lengthy and used set theoretic techniques. We are in debt to Professor Kent Fuller who communicated the present proof to us.

Proposition 4. Suppose $R$ is a semiperfect, right simple-injective ring with $\operatorname{soc}\left(R_{R}\right) \subseteq{ }^{\mathrm{ess}} R_{R}$. Given $n \geq 2$, if $\operatorname{soc}_{n}(R)$ is countably generated as a left $R$ module, then $J^{n-1} / J^{n}$ is finitely generated as a right $R$-module.

Proof. By Claims 3 and 4 of Proposition 1 , we have $S_{r}=S_{l} \subseteq{ }_{R}^{\text {ess }}{ }_{R}$. Write $S=S_{r}=S_{l}$. As $R / J$ is semisimple, $S=l(J)=r(J)$.

Claim. For all $n \geq 1, \operatorname{soc}_{n}\left(R_{R}\right)=\operatorname{soc}_{n}\left({ }_{R} R\right)=l\left(J^{n}\right)=r\left(J^{n}\right)$.

Proof. Suppose $\operatorname{soc}_{k}\left(R_{R}\right)=\operatorname{soc}_{k}\left({ }_{R} R\right)=l\left(J^{k}\right)=r\left(J^{k}\right)$. We have $\operatorname{soc}_{k+1}\left(R_{R}\right) \subseteq$ $l\left(J^{k+1}\right)$ because $\operatorname{soc}_{k+1}\left(R_{R}\right) / \operatorname{soc}_{k}(R)$ is right $R$-semisimple. On the other hand, if $a J^{k+1}=0$, then $a J \subseteq \operatorname{soc}_{k}(R)$ so $\left[a R+\operatorname{soc}_{k}(R)\right] / \operatorname{soc}_{k}(R)$ is right $R$-semisimple (as $R / J$ is semisimple). Hence $a R \subseteq \operatorname{soc}_{k+1}\left(R_{R}\right)$ and we have $\operatorname{soc}_{k+1}\left(R_{R}\right)=l\left(J^{k+1}\right)$. Similarly, $\operatorname{soc}_{k+1}\left({ }_{R} R\right)=r\left(J^{k+1}\right)$. Finally, $l\left(J^{k+1}\right)=r\left(J^{k+1}\right)$ follows easily from $l\left(J^{k}\right)=r\left(J^{k}\right)$. This proves the claim.

We now assert that if $I$ is a right ideal of $R$, every $R$-homomorphism $\varphi: I \rightarrow R$ with semisimple image is given by left multiplication by an element of $R$. Indeed, since $R$ is right finite dimensional by Proposition $1, \varphi(I)=\bigoplus_{i=1}^{n} S_{i}$ for simple right ideals $S_{i}$ of $R$. Let $\pi_{i}: \bigoplus_{i=1}^{n} S_{i} \rightarrow S_{i}$ be the projection for each $i$, and write $\varphi_{i}=\pi_{i} \circ \varphi$. Since $R$ is right simple-injective, there exist $t_{i} \in R$ such that $\varphi_{i}(a)=t_{i} a$ for all $a \in I$. If $t=\sum_{i=1}^{n} t_{i}$, then $\varphi(a)=t a$ for all $a \in I$.

With this it is straightforward to verify that

$$
\operatorname{hom}_{R}\left(J^{n-1} / J^{n}, R_{R}\right) \cong l_{R}\left(J^{n}\right) / l_{R}\left(J^{n-1}\right)
$$

for all $n \geq 2$. This implies that $J^{n-1} / J^{n}$ is finitely generated as a right $R$-module. Suppose not. Then, since $R$ has finitely many isomorphism classes of simple right modules, let $S^{(\mathbb{N})}$ be a direct summand of $J^{n-1} / J^{n}$ where $S$ is a simple right $R$-module and $S^{(\mathbb{N})}$ denotes a countable direct sum of copies of $S$. Now ${ }_{R} T=$ $\operatorname{hom}_{R}\left(S, R_{R}\right)$ is a simple left $R$-module because $R$ is right simple-injective and (by Proposition 1) right Kasch. Thus

$$
T^{\mathbb{N}}=\operatorname{hom}_{R}(S, R)^{\mathbb{N}} \cong \operatorname{hom}_{R}\left(S^{(\mathbb{N})}, R\right) \hookrightarrow \operatorname{soc}_{n}(R) / \operatorname{soc}_{n-1}(R)
$$


as a direct summand, where $T^{\mathbb{N}}$ is the direct product of countably many copies of $T$. But $T^{\mathbb{N}}$ has dimension $|T|^{|\mathbb{N}|}>|\mathbb{N}|$, according to a well known old theorem of Erdös and Kaplansky [6, Page 276], a contradiction.

Observe that the proof of Proposition 4 actually yields the following: If $R$ is a semiperfect, right simple-injective ring in which $\operatorname{soc}\left(R_{R}\right) \subseteq$ ess $R_{R}$ and $\operatorname{soc}_{2}(R)$ is generated on the left by $\chi$ elements, where $\chi$ is any ordinal number, then $\left(J / J^{2}\right)_{R}$ is generated by fewer than $\chi$ elements. For if this is not the case we can use the same argument to show that $\operatorname{soc}_{2}(R) / S$ contains a direct sum of $2^{\chi}>\chi$ simple modules, a contradiction. In particular, if $\operatorname{soc}_{2}(R)$ is generated on the left by $\omega$ elements (where $\omega$ is the first infinite ordinal), then $\left(J / J^{2}\right)_{R}$ is finitely generated.

Theorem 1. Suppose $R$ is a left perfect, right simple-injective ring. Then $R$ is quasi-Frobenius if and only if $\operatorname{soc}_{2}(R)$ is countably generated as a left $R$-module.

Proof. Proposition 4 and its proof show that $\operatorname{soc}_{2}\left(R_{R}\right)=\operatorname{soc}_{2}\left({ }_{R} R\right)$, and that $J / J^{2}$ is finitely generated as a right $R$-module. Hence $R$ is right artinian by Osofsky's theorem [15]. Then $R$ is right self-injective by Proposition 2. Thus $R$ is quasiFrobenius.

Theorem 2. Suppose $R$ is a left perfect right self-injective ring. Then $R$ is quasiFrobenius if and only if $\operatorname{soc}_{2}(R)$ is countably generated as a left $R$-module.

Remarks. Similar arguments give the following results:

(1) If $R$ is a semiperfect, right simple-injective ring with $\operatorname{soc}\left(R_{R}\right) \subseteq{ }^{\text {ess }} R_{R}$, and if $J / J^{2}$ is countably generated as a left $R$-module, then $\operatorname{soc}_{2}(R)$ is finitely generated as a right $R$-module.

(2) If $R$ is a left perfect, right self-injective ring, and if $J / J^{2}$ is countably generated as a left $R$-module, then $\operatorname{soc}_{2}(R)$ is finitely generated as a right $R$-module.

(3) If $R$ is a left and right perfect, right self-injective ring, and if $J / J^{2}$ is countably generated as a left $R$-module, then $R$ is quasi-Frobenius. (In fact, [8] shows that $R$ is quasi-Frobenius if $\operatorname{soc}_{2}(R)$ is finitely generated as a right $R$-module, so (2) applies.)

Example 1. If $R$ has zero right socle, then $R$ is right simple-injective. Hence the ring of integers is an example of a commutative, noetherian, simple-injective ring which is not self-injective (or principally injective).

Example 2. We construct an example of a left perfect, left simple-injective ring $S$ which is not right simple-injective (and hence not right self-injective). Let $R$ be a left perfect ring which is not right perfect. Since $R$ is left perfect, $\operatorname{soc}\left(R_{R}\right) \subseteq{ }^{\text {ess }} R_{R}$ and $\operatorname{soc}_{\lambda}\left(R_{R}\right)=R$ for some ordinal $\lambda$. Since $R$ is not right perfect (and hence not left semiartinian) $\operatorname{soc}_{\alpha}\left({ }_{R} R\right) \neq R$ for any ordinal $\alpha$. But $R$ is a set, so we have $\operatorname{soc}_{\beta}\left({ }_{R} R\right)=\operatorname{soc}_{\beta+1}\left({ }_{R} R\right)$ for some ordinal $\beta$. Let $S=R / \operatorname{soc}_{\beta}\left({ }_{R} R\right)$. Then $S$ is a left perfect ring with $\operatorname{soc}\left({ }_{S} S\right)=0$, and so $S$ is left simple-injective. But $S$ cannot be right simple-injective by Proposition 3. In particular, $S$ is not right self-injective.

A specific example is as follows: Let $F$ be a field and let $R$ be the ring of all lower triangular, countably infinite matrices over $F$ with only finitely many offdiagonal entries. Let $S$ be the $F$-subalgebra of $R$ generated by 1 and $J(R)$. Then $S$ is a left perfect, left simple-injective ring which is neither right perfect nor right simple-injective. Moreover, $S$ is not left self-injective because it is not left finite dimensional. 
Example 3 (Levy [13, page 115], see Hajarnavis-Norton [11, page 265]). Let $I=$ $\{x \mid x$ is real and $0 \leq x \leq 1\}$, let $K$ be a field, and let $R$ be the set of all formal sums of the form $\sum_{i \in I} a_{i} x^{i}$, where $x$ is a commuting indeterminate over $K, a_{i} \in K$, and all but a finite number of the $a_{i}$ are zero. Putting $x^{k}=0$ for $k>1, R$ becomes a commutative ring by defining addition and multiplication in the usual way. It can be verified that $R$ is a commutative, semiperfect, simple-injective, Kasch ring with essential socle, which satisfies $J^{2}=J$. However, $R$ is not self-injective.

Example 4 (Björk [5, page 70]). Let $p$ be a prime number, let $P$ be the field of $p$ elements, and let $K=P(x)$ be the field of rational functions with coefficients in $P$. Then $K^{p}=\left\{w^{p} \mid w \in K\right\}$ is a subfield of $K, f: w \mapsto w^{p}$ is an isomorphism $K \rightarrow K^{p}$, and $K^{p}$ is a $p$-dimensional vector space over $K$. If $A$ is a left vector space over $K$ with basis $\{e, x\}$, then $A$ is a ring with multiplication defined by: $e r=r e=r$ for all $r \in A, x^{2}=0$ and $f(w) x=x w$ for all $w \in K=K e$. The ring $A$ is clearly left and right artinian. Moreover, $A x=K x$ is the only proper left ideal of $A$, so that every left ideal is an annihilator; in particular, $A$ is right principally injective. But if $\left\{w_{1}, w_{2}, \ldots, w_{p}\right\}$ is a $K$-basis of $K^{p}$, then $w_{i} x A, i=1,2, \ldots, p$, are $p$ distinct minimal right ideals of $A$ so $A$ is not quasi-Frobenius. In particular, $A$ is not left or right simple-injective by Proposition 2.

Example 5 (Camillo [7, page 36]). Let $R$ be the ring generated over the field of two elements by variables $x_{1}, x_{2}, \ldots$ where $x_{i}^{3}=0=x_{i} x_{j}$ for all $i \neq j$, and $x_{i}^{2}=x_{j}^{2}$ for all $i$ and $j$. Then $R$ is a commutative, local, semiprimary, principally injective ring, but $R$ is not simple-injective by Proposition 2 because it is not artinian.

Conjecture. A left perfect, right simple-injective ring is right self-injective.

\section{REFERENCES}

1. P. Ara, and J. K. Park, On continuous semiprimary rings, Comm. Alg. 19 (1991), 1945-1957. MR 92g: 16006

2. E. P. Armendariz and J. K. Park, Selfinjective rings with restricted chain conditions, Arch. Math. 58 (1992), 24-33. MR 92m:16002

3. Y. Baba and K. Oshiro, On a Theorem of Fuller, J. Algebra, 154 (1993), 86-94. MR 94b: 16025

4. J. A. Beachy, On quasi-artinian rings, J. London Math. Soc. (2) 3 (1971), 449-452. MR 44:244

5. J.-E. Björk, Rings satisfying certain chain conditions, J. Reine Angew. Math. 245 (1970), 63-73. MR 43:3295

6. N. Bourbaki, "Algebra, Part I", Hermann/Addison Wesley, 1974, p. 400. MR 50:6689

7. V. Camillo, Commutative rings whose principal ideals are annihilators, Portugaliae Math. 46 (1) (1989), 33-37. MR 90e:13003

8. J. Clark and D. V. Huynh, A note on perfect selfinjective rings, Quart. J. Math. Oxford (2) 45 (1994), 13-17. MR 95a:16005

9. C. Faith, When self-injective rings are QF: A report on a problem, Centre Recerca Matemàtica Institut d'Estudis Catalans (Spain), 1990.

10. K. R. Fuller, On indecomposable injectives over artinian rings, Pacific J. Math. 29 (1969), 115-135. MR 40:186

11. C. R. Hajarnavis and N. C. Norton, On dual rings and their modules, J. Algebra 93 (1985), 253-266. MR 86i: 16016

12. M. Harada, On almost relative injective of finite length, preprint.

13. L. S. Levy, Commutative rings whose homomorphic images are self-injective, Pacific J. Math. 18 (1966), 149-153. MR 33:2663 
14. W. K. Nicholson and M. F. Yousif, Principally injective rings, J. Algebra 174 (1995), 77-93. MR 96i: 16005

15. B. L. Osofsky, A generalization of quasi-Frobenius rings, J. Algebra 4 (1966), 373-389. MR 34:4305; MR 36:6443

Department of Mathematics, University of Calgary, Calgary, Alberta, Canada T2N $1 \mathrm{~N} 4$

E-mail address: wknichol@acs.ucalgary.ca

Department of Mathematics, Ohio State University, Lima, Ohio 45804

E-mail address: yousif.1@osu.edu 